\title{
A DNA vaccine encoding a codon-optimized Human Papillomavirus Type 16 E6 gene enhances CTL response and anti-tumor activity
}

\author{
Cheng-Tao Lin $^{3}$, Ya-Chea Tsai ${ }^{1}$, Liangmei $\mathrm{He}^{1}$, Roanne Calizo ${ }^{1}$, Hung-Hsueh $\mathrm{Chou}^{3}$, \\ Ting-Chang Chang ${ }^{3}$, Yung-Kuei Soong ${ }^{3}$, Chien-Fu Hung ${ }^{1,2} * *$ \& Chyong-Huey Lai ${ }^{3}$ \\ ${ }^{1}$ Department of Pathology, Johns Hopkins Medical Institutions, CRB II Rm. 307, 1550 Orleans Street, \\ Baltimore, MD, 21231, USA; ${ }^{2}$ Department of Oncology, Johns Hopkins Medical Institutions, CRB II Rm. \\ 307, 1550 Orleans Street, Baltimore, MD, 21231, USA; ${ }^{3}$ Division of Gynecologic Oncology, Department of \\ Obstetrics and Gynecology, Chang Gung Memorial Hospital and Chung Gung University College of Medicine, \\ Taoyuan, Taiwan
}

Received 6 February 2006; accepted 28 March 2006

(C) 2006 National Science Council, Taipei

Key words: Human Papillomavirus (HPV), E6, E7, DNA vaccines

\begin{abstract}
Summary
The HPV oncoproteins E6 and E7 are consistently expressed in HPV-associated cancer cells and are responsible for their malignant transformation. Therefore, HPV E6 and E7 are ideal target antigens for developing vaccines and immunotherapeutic strategies against HPV-associated neoplasms. Recently, it has been demonstrated that codon optimization of the HPV-16 E7 gene resulted in highly efficient translation of E7 and increased the immunogenicity of E7-specific DNA vaccines. Since vaccines targeting E6 also represent an important strategy for controlling HPV-associated lesions, we developed a codon-optimized HPV-16 E6 DNA vaccine (pNGVL4a-E6/opt) and characterized the E6-specific CD8 ${ }^{+} \mathrm{T}$ cell immune responses as well as the protective and therapeutic anti-tumor effects in vaccinated C57BL/6 mice. Our data indicated that transfection of human embryonic kidney cells (293 cells) with pNGVL4a-E6/opt resulted in highly efficient translation of E6. In addition, vaccination with pNGVL4a-E6/opt significantly enhanced E6-specific $\mathrm{CD}^{+} \mathrm{T}$ cell immune responses in $\mathrm{C} 57 \mathrm{BL} / 6$ mice. Mice vaccinated with pNGVL4aE6/opt are able to generate potent protective and therapeutic antitumor effects against challenge with E6expressing tumor cell line, TC-1. Thus, DNA vaccines encoding a codon-optimized HPV-16 E6 may be a promising strategy for improving the potency of prophylactic and therapeutic HPV vaccines with potential clinical implications.
\end{abstract}

\section{Introduction}

Human Papillomavirus (HPV) infects large numbers of women worldwide and is present in more than $99 \%$ of all cervical cancers [1]. The HPV oncoproteins E6 and E7 are consistently expressed in HPV-associated cancer cells and are responsible for their malignant transformation. Therefore, HPV E6 and E7 are ideal target antigens for devel-

*To whom correspondence should be addressed. Fax:+1-443287-4295; E-mail: chung2@jhmi.edu oping vaccines and immunotherapeutic strategies against HPV-associated neoplasms. In the past, most HPV researchers have focused on E7, and therefore many E7-specific vaccines have been developed and characterized [2]. In comparison, relatively few vaccines targeting E6 have been reported. Since E6 represents another important target antigen for vaccines against HPV-associated lesions, there is strong interest in developing HPV vaccines employing E6.

DNA vaccines targeting the E6 antigen offer a potentially effective approach to prevent and 
control E6-expressing tumors. DNA vaccines represent a promising strategy for generating antigen-specific immunotherapy because of their simplicity, stability, safety, and capacity for repeated administration [3-6, 7]. Intradermal administration of DNA vaccines by means of a gene gun represents an efficient means of targeting dendritic cells, the most potent professional antigen-presenting cells, which are specialized to prime helper and killer T cells in vivo $[8,9]$. Using intradermally administered DNA vaccines, we have previously tested several strategies for modifying the properties of dendritic cells to enhance antigen presentation through the major histocompatibility complex (MHC) class I and class II pathways [10].

One strategy to enhance DNA vaccine potency is codon optimization. This strategy has been shown to enhance protein expression in several biological systems. This is accomplished by modification of codon composition to affect translational efficiency. Findings from several studies suggest that viral codon usage modified to resemble human gene codon usage can lead to an increased expression of the viral protein, as has been observed in the L1 and L2 capsid proteins of bovine papillomavirus type 1 as well as HPV-16 E7 protein $[11,12]$. The reported increase in HPV-16 E7 protein expression has also been shown to enhance the immunogenicity of E7-specific DNA vaccines. Liu et al. [13] previously demonstrated that a DNA vaccine encoding a codon-optimized HPV 16 E7 gene enhanced E7-specific cytotoxic T cell responses (CTL) and antitumor effects in vaccinated C57BL/6 mice. Similarly, Steinberg et al. [14] demonstrated that codon optimization of the HPV-16 E7 gene resulted in highly efficient translation of E7 as measured in transfection studies and increased the efficiency of CTL responses in vaccinated $\mathrm{C} 57 \mathrm{BL} / 6$ mice. These findings demonstrate that codon optimization has been effectively used to enhance the immunogenicity of E7-specific DNA vaccines.

Since both E7 and E6 are consistently expressed in HPV infections and HPV-associated malignancies, the codon optimization of the E6 gene represents a potential alternate approach to eliciting HPV-specific antitumor effects. Therefore, we developed a codon-optimized HPV-16 E6 DNA vaccine (pNGVL4a-E6/opt) and characterized the resultant E6-specific $\mathrm{CD}^{+} \mathrm{T}$ cell immune responses in vaccinated $\mathrm{C} 57 \mathrm{BL} / 6$ mice. We also assessed the protective and therapeutic anti-tumor effects generated by our DNA vaccine against challenge with an E6-expressing tumor cell line (TC-1) described previously $[15,16]$. Our findings indicate that transfection of 293 cells with DNA encoding a codon-optimized E6 can significantly increase the translation of E6. In addition, mice vaccinated with pNGVL4a-E6/opt are able to generate enhanced E6-specific $\mathrm{CD} 8{ }^{+} \mathrm{T}$ cell immune responses as well as potent protective and therapeutic antitumor effects against challenge with TC-1 tumor cells. Thus, DNA vaccines encoding a codon-optimized HPV-16 E6 may have clinical implications for improving the potency of prophylactic as well as therapeutic HPV vaccines.

\section{Methods}

Mice

C57BL/6 mice (6-8-week-old) were purchased from the National Cancer Institute (Frederick, MD). All animals were maintained under specific pathogen-free conditions at the Johns Hopkins Hospital (Baltimore, MD). All procedures were performed according to approved protocols and in accordance with recommendations for the proper care of laboratory animals.

Cells

Briefly, TC-1 cells were obtained by co-transformation of primary $\mathrm{C} 57 \mathrm{BL} / 6$ mouse lung epithelial cells with HPV-16 E6 and E7 and an activated ras oncogene as described previously [15]. The expression of E6 in TC-1 cells has also been characterized previously [16].

\section{DNA constructs}

To generate pNGVL4a-E6, E6 was isolated from pcDNA3-E6 [17] and cloned into pNGVL4a vector. To generate pNGVL4a-E6/opt, codonoptimized E6 synthesized by GenScript Corporation (Piscataway, $\mathrm{NJ}$ ) was cloned into EcoRI/ BamHI of pNGVL4a vector. The DNA and amino acid sequences of the wild-type E6 gene as well as the codon-optimized E6 were fully sequenced. 


\section{Western blot analysis}

Two-hundred and ninety three cells (from ATCC) were transiently transfected with pNGVL4a, pNGVL4a-E6, or pNGVL4a-E6/opt using Lipofectamine 2000 (Gibco, USA). At $24 \mathrm{~h}$ after transfection, the cells were lysed in Mammalian Protein Extraction Reagent (M-PER) (Pierce, Rockford, IL, USA) based on the manufacturer's instruction. Equal amounts ( $50 \mu \mathrm{g})$ of protein were loaded and separated by SDS-PAGE using a $10 \%$ polyacrylamide gel and blotted onto a PVDF membrane. After blocking, the membrane was incubated with rabbit anti-HPV-16 E6 polyclonal antibody for $2 \mathrm{~h}$ at room temperature, washed and incubated with horseradish peroxidase (HRP)-conjugated donkey anti-rabbit Ig (for E6) (Amersham Biosciences, UK). The blot was then washed and detected using chemiluminescence (ECL kit, Amersham, Arlington Heights, IL, USA). The other set of membrane was incubated with primary mouse anti-actin antibody (Sigma) and then incubated with horseradish peroxidase (HRP)-conjugated donkey anti-mouse Ig.

\section{$D N A$ vaccination}

DNA-coated gold particles were prepared, and gene gun particle-mediated DNA vaccination was performed, according to a protocol described previously [18]. Gold particles coated with pNGVL4a, pNGVL4a-E6 or pNGVL4a-E6/ opt were delivered to the shaved abdominal regions of mice by using a helium-driven gene gun (Bio-Rad Laboratories Inc., Hercules, Calif.) with a discharge pressure of $400 \mathrm{lb} / \mathrm{in} 2$. Mice were immunized with $2 \mu \mathrm{g}$ of the DNA vaccine and received two boosts with the same dose at 1-week interval. Splenocytes were harvested 1 week after the last vaccination.

\section{Intracellular cytokine staining and flow cytometry analysis}

Pooled splenocytes from the vaccinated mice were harvested 1 week after the last vaccination and incubated overnight with $1 \mu \mathrm{g} / \mathrm{ml}$ of the E6 peptide (aa50-57) [17] in the presence of GolgiPlug (BD Pharmingen, San Diego, CA, USA) $(1 \mu \mathrm{l} / \mathrm{ml})$. The stimulated splenocytes were then washed once with FACScan buffer and stained with phycoerythrin-conjugated monoclonal rat anti-mouse CD8a (clone 53.6.7). Cells were subjected to intracellular cytokine staining using the Cytofix/Cytoperm kit according to the manufacturer's instruction (BD Pharmingen, San Diego, CA, USA). Intracellular IFN- $\gamma$ was stained with FITC-conjugated rat anti-mouse IFN $-\gamma$. All antibodies were purchased from BD Pharmingen. Flow cytometric analysis was performed using FACSCalibur with CELLQuest software (BD Biosciences, Mountain View, CA, USA).

\section{In vivo tumor protection experiment}

For in vivo tumor protection experiment, C57BL $/ 6$ mice (five per group) were immunized via gene gun with $2 \mu \mathrm{g}$ of pNGVL4a, pNGVL4a-E6, or pNGVL4a-E6/opt. Mice were boosted twice with the same regimen as that used in the first vaccination. One week after the last vaccination, mice were challenged with $5 \times 10^{4}$ TC- 1 tumor cells/ mouse subcutaneously in the right leg and monitored once a week by inspection and palpation.

\section{In vivo tumor treatment experiment}

For in vivo tumor treatment experiment, $1 \times 10^{4}$ TC-1 tumor cells were injected into 5-8-week-old C57BL/6 mice (five per group) via tail vein. After 3 days, the mice were immunized with the DNA vaccines as described above. After 1 week, these mice were boosted once with the same immunization regimen. At 28 days after tumor challenge, the mice were killed, and the lung tumor nodules were counted. Data are expressed as the mean number of lung nodules \pm standard error (s.e.). The data shown here represent one of the two experiments performed.

\section{Statistical analysis}

All data expressed as means \pm s.e. are representative of at least two different experiments. Data for intracellular cytokine staining with flow cytometry analysis were evaluated by ANOVA. Comparisons between individual data points were made using a Student's $t$-test. For statistical analysis of the tumor protection experiment, we used Kaplan-Meier analysis. 


\section{Results}

Transfection of cells with DNA encoding a codon-optimized HPV-16 E6 gene significantly increased E6 expression

To verify that human embryonic kidney cells (293 cells) transfected with codon-optimized E6 DNA are capable of expressing HPV-16 E6, 293 cells were transfected with an empty DNA construct (pNGVL4a), DNA encoding a wild-type E6 protein (pNGVL4a-E6) or DNA encoding a codon-optimized E6 protein (pNGVL4a-E6/opt). Western blot analysis was performed with $50 \mu \mathrm{g}$ of cell lysates $24 \mathrm{~h}$ after transfection. Actin was used as a control to indicate that equal amounts of cell lysates were loaded. As seen in Figure 1, Western blot analysis revealed a band in cell lysates from pNGVL4a-E6/opt-transfected cells but not from pNGVL4a- or pNGVL4a-E6-transfected cells.

Since the E6 expression in cells transfected with pNGVL4a-E6 was barely detectable, it was difficult to estimate the fold increase in E6 expression resulting from the transfection of cells with

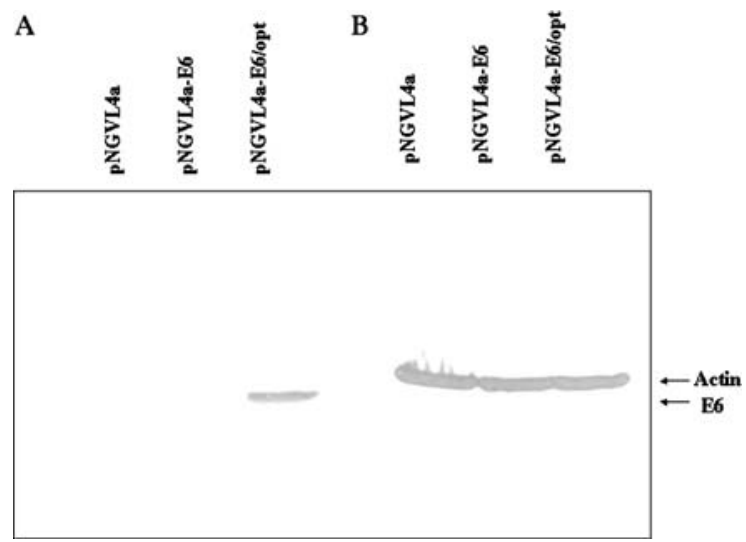

Figure 1. Western blot analysis to detect the expression of HPV-16 E6 protein in human embryonic kidney cells (293) transfected with various DNA constructs. 293 cells were transfected with $2 \mu \mathrm{g}$ of an empty DNA construct (pNGVL4a), DNA encoding a wild-type E6 gene (pNGVL4aE6) or DNA encoding a codon-optimized E6 gene (pNGVL4a-E6/opt). Western blot analysis was performed with $50 \mu \mathrm{g}$ of cell lysates $24 \mathrm{~h}$ after transfection. E6 expression was detected using rabbit anti-HPV16-E6 polyclonal antibody and HRP-conjugated donkey anti-rabbit Ig for E6 (Amersham Biosciences). Actin was used as a control to indicate that equal amounts of cell lysates were loaded. (a) Detection of E6 expression in cells transfected with pNGVL4a, pNGVL4a-E6 or pNGVL4a-E6/opt. (b) Detection of actin expression in cells transfected with pNGVL4a, pNGVL4a-E6 or pNGVL4a-E6/opt.
pNGVL4a-E6/opt. The fact that a significant level of E6 expression was detected in pNGVL4a-E6/ opt-transfected cells indicates that codon optimization of the E6 gene can markedly enhance E6 expression.

Vaccination with pNGVL4a-E6/opt generates E6-specific $C D 8^{+} T$-cell immune responses in $C 57 B L / 6$ mice

Since we have successfully generated a codonoptimized E6 DNA vaccine (pNGVL4a-E6/opt) that enhances the expression of HPV-16 E6, we next explored whether $\mathrm{C} 57 \mathrm{BL} / 6$ mice vaccinated with pNGVL4a-E6/opt could generate E6-specific $\mathrm{CD} 8{ }^{+}$T-cell immune responses. We vaccinated mice thrice with pNGVL4a, pNGVL4a-E6 or pNGVL4a-E6/opt using the same dose and vaccination regimen for each construct. To assess the quantity of E6-specific $\mathrm{CD}^{+} \mathrm{T}$ cell precursors generated by each of the various DNA constructs, we performed intracellular cytokine staining followed by flow cytometric analysis using splenocytes derived from the immunized mice at 7 days after the last (third) vaccination. As shown in Figure 2, mice vaccinated with pNGVL4a-E6/opt generated the highest number of E6-specific CD ${ }^{+}$ T cells $\left(273 \pm 24 / 3 \times 10^{5}\right.$ splenocytes $)$ compared to mice vaccinated with pNGVL4a $\left(4 \pm 2 / 3 \times 10^{5}\right.$ splenocytes) or pNGVL4a-E6 $\left(6.5 \pm 2 / 3 \times 10^{5}\right.$ splenocytes) (one-way analysis of variance (ANOVA), $p<0.01)$. Thus, our data suggest that vaccination with codon-optimized E6 DNA can generate more potent E6-specific $\mathrm{CD}^{+} \mathrm{T}$ cell immune responses compared to wild-type E6 DNA vaccination.

Vaccination with pNGVL4a-E6/opt generates protective antitumor effects against challenge with an HPV-16 E6-expressing tumor cell line, TC-1

To determine whether the pNGVL4a-E6/opt vaccine can also provide protection against E6-expressing tumors in vaccinated mice, we immunized C57BL/6 mice thrice with pNGVL4a, pNGVL4a-E6 or pNGVL4a-E6/opt using the same dose and vaccination regimen for each construct. After the last vaccination, the mice were subcutaneously challenged with an HPV-16 E6-expressing tumor cell model, TC-1 [15] and monitored for evidence of tumor growth by inspection and 

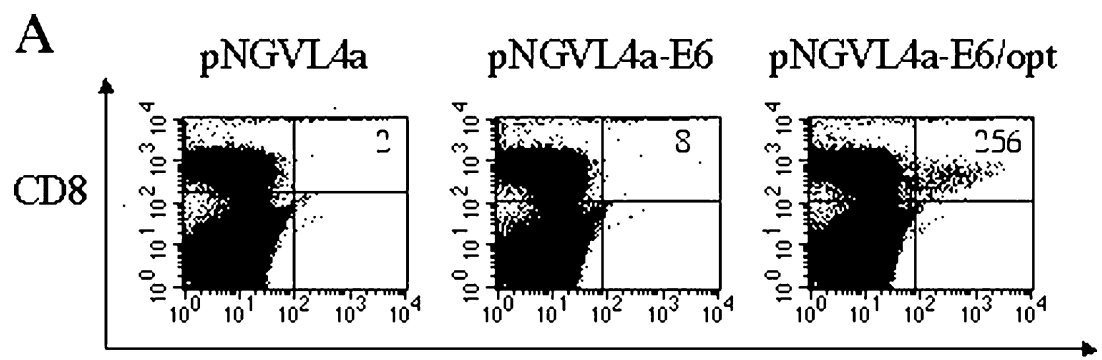

IFN- $\gamma$

B

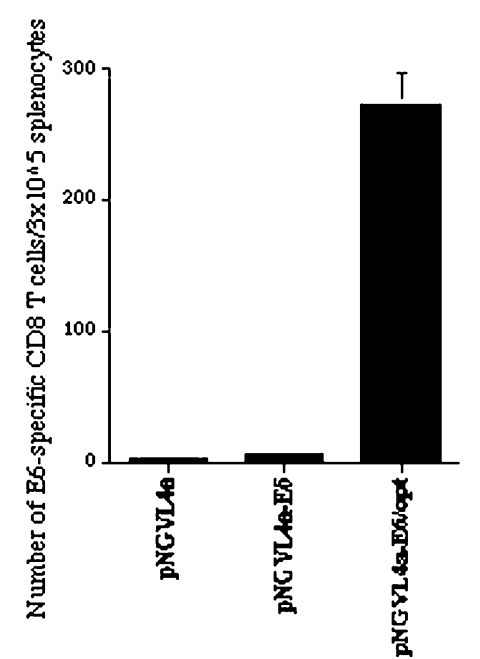

Figure 2. Intracellular cytokine staining with flow cytometric analysis to determine the number of E6-specific $\mathrm{CD} 8^{+} \mathrm{T}$ cells in mice after immunization with the various DNA constructs. C57BL/6 mice (5 per group) were vaccinated thrice with $2 \mu \mathrm{g}$ pNGVL4a, pNGVL4a-E6 or pNGLV4a-E6/opt DNA constructs at 1-week interval. Splenocytes were harvested 7 days after the last vaccination, cultured in vitro with MHC class I-restricted E6 (aa50-57) peptide overnight and stained for CD8 ${ }^{+} \mathrm{T}$ cells. (a) Representative figure of the flow cytometry data. (b) Bar graph depicting the number of E6-specific CD8 ${ }^{+} \mathrm{T}$ cell precursors per $3 \times 10^{5}$ splenocytes (means \pm s.e.).

palpation twice a week. As shown in Figure 3,60\% of mice immunized with codon-optimized E6 DNA remained tumor-free 63 days after TC- 1 tumor challenge. In contrast, $100 \%$ of the mice immunized with the empty DNA construct or DNA encoding wild-type E6 exhibited tumor growth within 63 days after TC- 1 tumor challenge (Kaplan-Meier Analysis, $p<0.01$ ). These data suggest that DNA encoding a codon-optimized E6 gene can generate enhanced protective effects against E6-expressing tumors in mice compared to DNA encoding a wild-type E6 gene.

Treatment with pNGVL4a-E6/opt generates therapeutic anti-tumor effects against TC-1 tumor cells

The therapeutic potential of each vaccine was assessed by performing an in vivo tumor treatment experiment using a previously described lung hematogeneous spread model. Mice were challenged with TC-1 tumor cells via tail vein injection followed by treatment with DNA vaccines. As shown in Figure 4, C57BL/6 mice treated with pNGVL4a-E6/opt exhibited the lowest mean number of pulmonary tumor nodules $(17 \pm 6)$, significantly lower than mice treated with pNGVL4a $(120 \pm 22)$ or pNGVL4a-E6 (108 \pm 27$)$ (one-way analysis of variance (ANOVA), $p<0.01$ ). These data indicate that treatment with pNGVL4a-E6/ opt generates the most potent antitumor effect against E6-expressing tumors compared to treatment with pNGVL4a or pNGVL4a-E6.

\section{Discussion}

In the current study, we investigated the feasibility of using a codon-optimized HPV-16 E6 gene for 


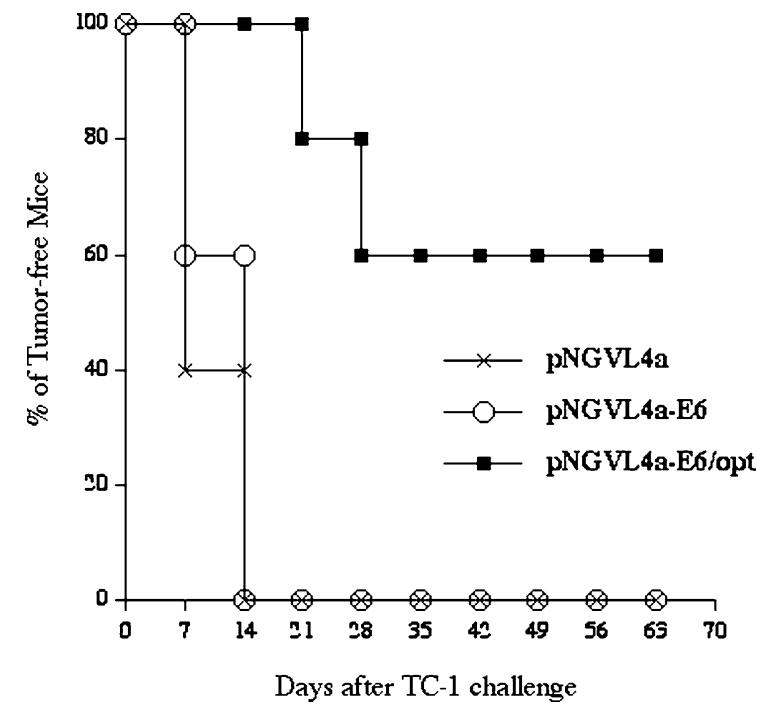

Figure 3. In vivo tumor protection experiment using HPV-16 E6-expressing tumor cell model, TC-1. C57BL/6 mice (5 per group) were immunized thrice with $2 \mu \mathrm{g}$ pNGVL4a, pNGVL4a-E6 or pNGLV4a-E6/opt DNA vaccines at 1-week interval. At 7 days after the last vaccination, the mice were challenged subcutaneously with $5 \times 10^{4} \mathrm{TC}-1$ cells/mouse and monitored for evidence of tumor growth by inspection and palpation twice a week. The graph shows the percentage of tumor-free mice days after the tumor challenge.

enhancing the potency of a DNA vaccine intended to prevent or control HPV-associated neoplasms. Codon optimization of the E6 gene resulted to increased E6 expression and strong E6-specific $\mathrm{CD} 8{ }^{+} \mathrm{T}$ cell immune responses in C57BL/6 mice. Furthermore, vaccination with DNA encoding a codon-optimized E6 gene generated strong protective anti-tumor effects against challenge with E6-expressing tumors and therapeutic effects against established E6-expressing tumors. Altogether, these findings suggest that codon optimization of the E6 gene may be a promising strategy to prevent and control HPV-associated neoplasms.

The observed enhancement in E6 protein expression by codon optimization is likely contributed by the increased translation of E6 mRNA, although we cannot completely exclude the contribution from better RNA transcription or stability. In one study, the ratios of mRNAs from Cos-1 cells transfected with wildtype E7 or codon-optimized E7 normalized using $\beta$-actin as internal standard transcription were found to be 1:1.3 (wild-type E7: codon-optimized E7), but the E7 protein expression was dramatically different. Thus, it was concluded that the

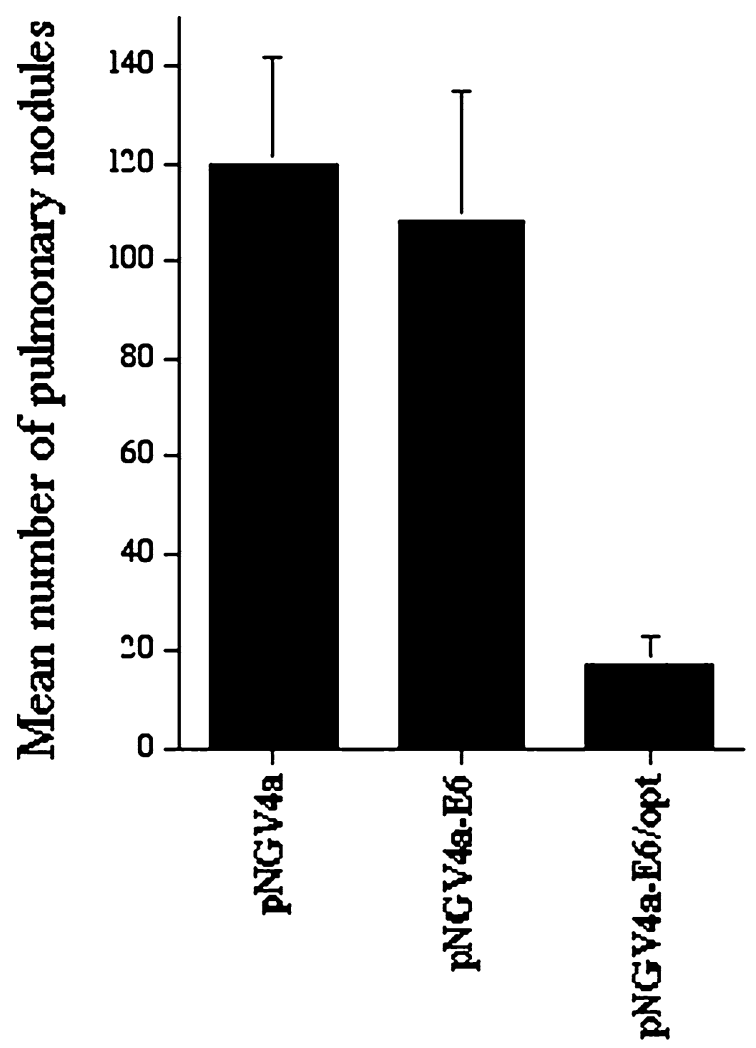

Figure 4. In vivo tumor treatment experiment to compare the antitumor effect generated by various DNA constructs in mice. C57BL/6 mice (five per group) were challenged with $1 \times 10^{4}$ cells/mouse TC-1 tumor cells by tail vein injection. After 3 days, the mice were primed and boosted with $2 \mu \mathrm{g}$ pNGVL4a, pNGVL4a-E6 or pNGLV4a-E6/opt at 1-week interval. Data are expressed as the mean number of lung nodules \pm s.e. The data shown here are from one of two experiments performed.

observed enhancement in E7 expression by codon optimization was a consequence of improved E7 mRNA translation, rather than increased E7 mRNA transcription or better E7 mRNA stability [13].

To date, studies have not fully addressed the underlying mechanisms accounting for limited HPV protein expression without codon optimization. One explanation may derive from the differences in codon usage between HPV and human genes. HPV-16 genes usually have a high rate of A or $\mathrm{T}$ content at the third sequence, which infrequently occurs in human genes. In comparison to humans, HPV-16 genes also have a markedly lower $\mathrm{G}+\mathrm{C}$ content at the third sequence [11]. Previous experiments modifying the codon compositions of some viruses to resemble typical 
mammalian genes demonstrated efficient viral protein translation $[12,19,20]$. It is conceivable that the divergence of viral codon usage from mammalian gene codon usage results in insufficient viral tRNAs for viral protein translation [11, 12, 21-23]. Thus, the codon optimization of viral gene can facilitate the usage of mammalian tRNA for viral protein translation.

While enhanced E6 translation may account for the increase in E6 protein expression, leading to better immune responses, it is also be possible that cytokine release induced by immunostimulatory $\mathrm{CpG}$ motifs may have enhanced the generation of CTL responses. It has been shown that linkage of these motifs to plasmid DNA or protein immunogens enhances innate immune response characterized by cytokine release and boosts antigen-specific immune responses [13, 24]. Since codon optimization may increase number of typical immunostimulatory $\mathrm{CpG}$ motifs within the E6 gene, we cannot exclude the possibility that these motifs may have also promoted the antigen-specific immune responses.

The encouraging results from this study suggest that codon optimization may also be used to enhance the potency of DNA vaccines targeting multiple tumor antigens. For instance, since E6 and E7 are both important for the malignant transformation of HPV-associated neoplasia and are coexpressed in nearly all HPV-associated tumors, both antigens represent ideal candidates for codon optimization. We have previously demonstrated that coadministration of CRT/E6 and CRT/E7 vaccines generates a better anti-tumor effect compared to the administration of either CRT/E6 DNA or CRT/E7 DNA [25]. In view of this observation, it is likely that a combination of DNA vaccines encoding codon-optimized E6 and codon-optimized E7 may provide more potent antitumor effects than vaccination with codon-optimized E6 alone.

In summary, our data suggest that codon optimization of E6 leads to the increased expression of E6, resulting to the enhancement of DNA vaccine potency by generating stronger $\mathrm{CD} 8^{+} \mathrm{T}$ cell immune responses as well as more potent protective and therapeutic antitumor effects. These observations suggest that it may be desirable to consider evaluating the efficacy of DNA vaccines simultaneously encoding multiple codon-optimized genes, such as E6 and E7. In addition, the use of codon optimization in conjunction with other vaccination strategies represents a potential opportunity for future clinical translation of powerful prophylactic and therapeutic treatments for controlling HPV-associated lesions. The safety issue associated with the clinical translation of DNA vaccines encoding the oncogenic antigens E6 and E7 can be overcome by using mutant, attenuated versions of E6 and E7.

\section{Acknowledgements}

We gratefully acknowledge Drs. T.-C. Wu and Richard Roden for helpful discussions. We would also like to thank Dr. Ralph Hruban and David Boyd for critical review of this paper. This work was supported by Flight Attendant Medical Research Institute and Grants CMRPG 32088 and 34022 from the Chang Gung Memorial Hospital.

\section{References}

1. Walboomers J.M., Jacobs M.V., Manos M.M., Bosch F.X., Kummer J.A., Shah K.V., Snijders P.J., Peto J., Meijer C.J. and Munoz N., Human papillomavirus is a necessary cause of invasive cervical cancer worldwide. J. Pathol. 189: 12-19, 1999.

2. Ling M., Kanayama M., Roden R. and Wu T.-C., Preventive and therapeutic vaccines for HPV-associated cervical cancers. J. Biomed. Sci. 7: 341-356, 2000.

3. Donnelly J.J., Ulmer J.B., Shiver J.W. and Liu M.A., DNA vaccines. Annu. Rev. Immunol. 15: 617-648, 1997.

4. Moniz M., Ling M., Hung C.F. and Wu T.C., HPV DNA vaccines. Front. Biosci. 8: d55-d68, 2003.

5. Pardoll D.M. and Beckerleg A.M., Exposing the immunology of naked DNA vaccines. Immunity 3: 165-169, 1995.

6. Robinson H.L. and Torres C.A., DNA vaccines. Semin. Immunol. 9: 271-283, 1997.

7. Shedlock D.J. and Weiner D.B., DNA vaccination: antigen presentation and the induction of immunity. J. Leukoc. Biol. 68: 793-806, 2000.

8. Condon C., Watkins S.C., Celluzzi C.M., Thompson K. and Falo L.D. Jr, DNA-based immunization by in vivo transfection of dendritic cells. Nat. Med. 2: 1122-1128, 1996.

9. Porgador A., Irvine K.R., Iwasaki A., Barber B.H., Restifo N.P. and Germain R.N., Predominant role for directly transfected dendritic cells in antigen presentation to CD8+ T cells after gene gun immunization. J. Exp. Med. 188: 1075-1082, 1998.

10. Hung C.F. and Wu T.C., Improving DNA vaccine potency via modification of professional antigen presenting cells. Curr. Opin. Mol. Ther. 5: 20-24, 2003.

11. Cid-Arregui A., Juarez V. and zur Hausen H., A synthetic E7 gene of human papillomavirus type 16 that yields 
enhanced expression of the protein in mammalian cells and is useful for DNA immunization studies. J. Virol. 77: 4928-4937, 2003.

12. Zhou J., Liu W.J., Peng S.W., Sun X.Y. and Frazer I., Papillomavirus capsid protein expression level depends on the match between codon usage and tRNA availability. J. Virol. 73: 4972-4982, 1999.

13. Liu W.J., Gao F., Zhao K.N., Zhao W., Fernando G.J., Thomas R. and Frazer I.H., Codon modified human papillomavirus type 16 E7 DNA vaccine enhances cytotoxic T-lymphocyte induction and anti-tumour activity. Virology 301: 43-52, 2002.

14. Steinberg T., Ohlschlager P., Sehr P., Osen W. and Gissmann L., Modification of HPV 16 E7 genes: correlation between the level of protein expression and CTL response after immunization of C57BL/6 mice. Vaccine 23 : 1149-1157, 2005.

15. Lin K.-Y., Guarnieri F.G., Staveley-O'Carroll K.F., Levitsky H.I., August T., Pardoll D.M. and Wu T.-C., Treatment of established tumors with a novel vaccine that enhances major histocompatibility class II presentation of tumor antigen. Cancer Res. 56: 21-26, 1996.

16. He Z., Wlazlo A.P., Kowalczyk D.W., Cheng J., Xiang Z.Q., Giles-Davis W. and Ertl H.C., Viral recombinant vaccines to the E6 and E7 antigens of HPV-16. Virology 270: 146-161, 2000.

17. Peng S., Hung C.-F., Trimble C., He L., Yeatermeyer J., Boyd D. and Wu T.-C., Development of a DNA vaccine targeting HPV-16 oncogenic protein E6. J. Virol. 78: 8468-8476, 2004.
18. Chen C.-H., Wang T.-L., Hung C.-F., Yang Y., Young R.A., Pardoll D.M. and Wu T.-C., Enhancement of DNA vaccine potency by linkage of antigen gene to an HSP70 gene. Cancer Res. 60: 1035-1042, 2000.

19. Haas J., Park E.C. and Seed B., Codon usage limitation in the expression of HIV-1 envelope glycoprotein. Curr. Biol. 6: 315-324, 1996

20. Karlin S., Blaisdell B.E. and Schachtel G.A., Contrasts in codon usage of latent versus productive genes of EpsteinBarr virus: data and hypotheses. J. Virol. 64: 4264-4273, 1990.

21. Grantham R., Gautier C. and Gouy M., Codon frequencies in 119 individual genes confirm consistent choices of degenerate bases according to genome type. Nucleic Acids Res. 8: 1893-1912, 1980.

22. Wain-Hobson S., Nussinov R., Brown R.J. and Sussman J.L., Preferential codon usage in genes. Gene 13: 355-364, 1981.

23. Ikemura T., Correlation between the abundance of yeast transfer RNAs and the occurrence of the respective codons in protein genes. Differences in synonymous codon choice patterns of yeast and Escherichia coli with reference to the abundance of isoaccepting transfer RNAs. J. Mol. Biol. 158: 573-597, 1982.

24. Klinman D.M., Barnhart K.M. and Conover J., CpG motifs as immune adjuvants. Vaccine 17: 19-25, 1999.

25. Peng S., Tomson T.T., Trimble C., He L., Hung C.F. and $\mathrm{Wu}$ T.C., A combination of DNA vaccines targeting human papillomavirus type 16 E6 and E7 generates potent antitumor effects. Gene Ther. 2006 13:257-265. 